\title{
An Overview on Molecular Basis of Genetic Recombination
}

\author{
Mamta Nehra $^{1}$, Rajesh Kumar Sharma ${ }^{2}$ and Mukesh Choudhary ${ }^{3}$ \\ ${ }^{1}$ G B Pant University of Agriculture and Technology, Pantnagar- 263145, India \\ ${ }^{2}$ ICAR-Indian Agricultural Research Institute, New Delhi-110012, India \\ ${ }^{3}$ ICAR-Indian Institute of Maize Research, Ludhiana- 141 004, India \\ *Corresponding author
}

\begin{tabular}{l} 
K e y w o r d s \\
$\begin{array}{l}\text { Recombination, } \\
\text { Holliday model, } \\
\text { DSB model, } \\
\text { RecBCD enzyme. }\end{array}$ \\
\hline Article Info \\
$\begin{array}{l}\text { Accepted: } \\
12 \text { March } 2017 \\
\text { Available Online: } \\
\text { 10 April } 2017\end{array}$
\end{tabular}

\section{Introduction}

Recombination is the production of new DNA molecule(s) from two parental DNA molecules or different segments of the same DNA molecule. At least four types of naturally occurring recombination have been identified in living organisms. General or homologous recombination occurs between DNA molecules of very similar sequence, such as homologous chromosomes in diploid organisms. General recombination can occur throughout the genome of diploid organisms, using one or a small number of common enzymatic pathways.
Illegitimate or non homologous recombination occurs in regions where no large-scale sequence similarity is apparent, e.g. translocations between different chromosomes or deletions that remove several genes along a chromosome. Another type of recombination, called site specific recombination, can alter gene order and also add new information to the genome. Sitespecific recombination moves specialized nucleotide sequences, called mobile genetic elements, between non homologous sites within a genome. The movement can occur 
between two different positions in a single chromosome, as well as between two different chromosomes. Sitespecific recombination can proceed via either of two distinct mechanisms, each of which requires specialized recombination enzymes and specific DNA sites (1) Transpositional site-specific recombination usually involves breakage reactions at the ends of the mobile DNA segments embedded in chromosomes and the attachment of those ends at one of many different non homologous target DNA sites. It does not involve the formation of heteroduplex DNA. (2) Conservative sitespecific recombination involves the production of a very short heteroduplex joint, and it therefore requires a short DNA sequence that is the same on both donor and recipient DNA molecules.

The process of homologous genetic recombination is essential to all organisms. It is important for the generation of genetic diversity, the maintenance of genomic integrity, and the proper segregation of chromosomes. The predictable assortment of genes by homologous recombination underlies the basic principles of genetics. Yet despite the importance of this central biological process, the biochemical details of the molecular mechanism by which segments of DNA are exchanged between chromosomal homologs are unclear. Part of the explanation for this uncertainty lies in the biochemical uniqueness and complexity of the recombination process. In contrast to the mechanisms of many other transactions involving nucleic acids (e.g., replication, transcription, and translation), which result in net macromolecular biosynthesis, the simplest models of homologous recombination mandated little or no DNA synthesis; thus, biochemical assays for components of the recombination apparatus were not easily forthcoming. Furthermore, the isolation and characterization of bonafide intermediates of the recombination process proved impractical because of their complexity, liability, and low abundance, eliminating that potentially informative avenue of investigation. Finally, genetic analysis uncovered the complexity of the recombination processes, demonstrating the involvement of a multitude of genes. For example, in both the prokaryote Escherichia coli and the eukaryote Saccharomyces cerevisiae, dozens of genes were identified as either essential or important to genetic recombination. In most cases, the genes identified were not involved in other DNA metabolic processes but, instead, were shown to be uniquely important to recombination or recombinational repair. The genetic disclosure of redundant functions served to underscore the intricacy of the recombination process and to hinder identification of the role of each gene product in the recombination mechanism (Kowalczykowski et al., 1994).

\section{Holliday Model for General Recombination: Single Strand Invasion}

In 1964, Robin Holliday proposed a model that accounted for heteroduplex formation and gene conversion during recombination. It illustrates the critical steps of pairing of homologous duplexes, formation of a heteroduplex, formation of the recombination joint, branch migration and resolution (Fig. 1).

The steps in the Holliday Model are-

1. Two homologous chromosomes, each composed of duplex DNA, are paired with similar sequences adjacent to each other.

2. An endonuclease nicks at corresponding regions of homologous strands of the paired duplexes.

3. The nicked ends dissociate from their complementary strands and each single 
strand invades the other duplex. This occurs in a reciprocal manner to produce a heteroduplex region derived from one strand from each parental duplex.

4. DNA ligase seals the nicks. The result is a stable joint molecule, in which one strand of each parental duplex crosses over into the other duplex. This X-shaped joint is called a Holliday intermediate or Chi structure.

5. Branch migration then expands the region of heteroduplex. The stable joint can move along the paired duplexes, feeding in more of each invading strand and extending the region of heteroduplex.

The recombination intermediate is then resolved by nicking a strand in each duplex and ligation.

Resolution can occur in either of two ways, only one of which results in an exchange of flanking markers after recombination. The two modes of resolution can be visualized by rotating the duplexes so that no strands cross over each other in the illustration. In the "horizontal" mode of resolution, the nicks are made in the same DNA strands that were originally nicked in the parental duplexes. After ligation of the two ends, this produces two duplex molecules with a patch of heteroduplex, but no recombination of flanking regions. In contrast, for the "vertical" mode of resolution, the nicks are made in the other strands, i.e. those not nicked in the original parental duplexes. Ligation of these two ends also leaves a patch of heteroduplex, but additionally causes recombination of flanking regions.

Although the original Holliday model accounted for many important aspects of recombination (all that were known at the time), some additional information requires changes to the model. For instance, the Holliday model treats both duplexes equally; both are the invader and the target of the strand invasion. Also, no new DNA synthesis is required in the Holliday model. However, subsequent work showed that one of the duplex molecules is the used preferentially as the donor of genetic information. These ideas have been incorporated into a new model of recombination involving double strand breaks in the DNAs.

\section{Double-strand-break model for recombination}

A new model for recombination from Jack Szostak and colleagues given in1983 (Szostak et al., 1983), it is called the double-strandbreak model. New features in this model (contrasting with the Holliday model) are initiation at double-strand breaks, nuclease digestion of the aggressor duplex, new synthesis and gap repair. However, the fundamental Holliday junction, branch migration and resolution are retained, albeit with somewhat greater complexity because of the additional numbers of Holliday junctions.

The steps in the double-strand-break model up to the formation of the joint molecules are diagrammed in figure 2 .

1. An endonuclease cleaves both strands of one of the homologous DNA duplexes. This is the aggressor duplex, since it initiates the recombination. It is also the recipient of genetic information, as will be apparent as we go through the model.

2. The cut is enlarged by an exonuclease to generate a gap with $3^{\prime}$ single-stranded termini on the strands.

3. One of the free 3 ' ends invades a homologous region on the other duplex called the donor duplex. The formation of 
heteroduplex also generates a D-loop (a displacement loop), in which one strand of the donor duplex is displaced.

4. The D-loop is extended as a result of repair synthesis primed by the invading $3^{\prime}$ end. The D-loop eventually gets large enough to cover the entire gap on the aggressor duplex, i.e. the one initially cleaved by the endonuclease. The newly synthesized DNA uses the DNA from the invaded DNA duplex as the template, so the new DNA has the sequence specified by the invaded DNA.

5. When the displaced strand from the donor extends as far as the other side of the gap on the recipient, it will anneal with the other 3' single stranded end at that end of the gap. The displaced strand has now filled the gap on the aggressor duplex, donating its sequence to the duplex that was initially cleaved. Repair synthesis catalyzed by DNA polymerase converts the donor D-loop to duplex DNA. The duplex that was initially invaded serves as the donor duplex; i.e. it provides genetic information during this phase of repair synthesis. Conversely, the aggressor duplex is the recipient of genetic information. Note that the single strand invasion models predict the opposite, where the initial invading strand is the donor of the genetic information.

6. DNA ligase will seal the nicks, one on the left side and the other on the right side. Although the latter is between a strand on the bottom duplex and a strand on the top duplex, it is equivalent to the ligation in the first nick. In both cases, sealing the nick forms a Holliday junction.

At this point, the recombination intermediate has two recombinant joints (Holliday junctions). The original gap in the aggressor duplex has been filled with DNA donated by the invaded duplex. The filled gap is now flanked by heteroduplex. The heteroduplexes are arranged asymmetrically, with one to the left of the filled gap on the aggressor duplex and one to the right of the filled gap on the donor duplex. Branch migration can extend the regions of heteroduplex from each Holliday junction. Each joint can be resolved horizontally or vertically. The key factor is whether the joints are resolved in the same mode or sense (both horizontally or both vertically) or in different modes.

If both joints are resolved the same sense, the original duplexes will be released, each with a region of altered genetic information that is a "footprint" of the exchange event. That region of altered information is the original gap, plus or minus the regions covered by branch migration. For instance, if both joints are resolved by cutting the originally cleaved strands ("horizontally" in our diagram of the Holliday model), then you have no crossover at either joint. If both joints are resolved by cleaving the strands not cut originally ("vertically" in our diagram of the Holliday model), then you have a crossover at both joints. This closely spaced double crossover will produce no recombination of flanking markers.

\section{Enzymes required for recombination in $E$.} coli

At least 25 different proteins are involved in all types of homologous recombination in $\mathrm{E}$. coli; these include the RecA, RecBCD, RecF, RecG, RecJ, RecN, RecO, RecQ, RecR, RuvAB, RuvC, PriA and SSB proteins, DNA polymerases, DNA topoisomerases and DNA ligase, as well as the cis-acting recombination hotspot $x$ (Kowalczykowski et al.,1994). Three different pathways have been characterized that differ in the steps used to generate the invading single strand of DNA. 
All three pathways use RecA for homologous pairing and strand exchange, RuvA and RuvB for branch migration, and RuvC and DNA ligase for resolution.

The E. coli RecBCD enzyme is a multifunctional protein complex $(330 \mathrm{kDa})$ containing three subunits, the products of the recB, recC, and recD genes. This enzyme displays four distinct activities: nuclease, helicase, ATPase, and site-specific recognition of the DNA regulatory sequence chi (crossover hotspot instigator, $x$ ). Originally identified as exonuclease $\mathrm{V}$, the RecBCD enzyme is responsible for the seemingly disparate functions of DNA degradation and repair of the bacterial chromosome. Under optimal conditions, the RecBCD enzyme is a highly processive helicase, unwinding an average of 30 kilobases per binding event at a rate of 10001500 base pairs per second (Fig. 3) (Roman et al., 1992). A blunt double stranded DNA (dsDNA) end is the preferred substrate for initiation of unwinding, but single-stranded DNA (ssDNA) tails of less than 25 nucleotides can also serve as initiation sites.

During unwinding, either ssDNA loop-tails, or twin loops of ssDNA are formed, which extend from the RecBCD enzyme complex (Taylor and Smith, 1980). Atphysiological temperature (378C), the loops grow at a rateof about 100 nucleotides per second. The presence of the loop-tail or twin-loop structures implies that there are at least two translocating domains in the holoenzyme: a more rapidly moving domain(s) containing the helicase activity and a slower domain(s) that translocates along the ssDNA produced. Thus, as the dsDNA substrate is processed, ssDNA loop(s) form between these domains. E. coli single stranded DNA-binding protein (SSB) favours production of the loop-tail structure with the loop being createdfrom the DNA strand terminating with a 3' hydroxyl at the point of entry of the RecBCD enzyme, presumably by binding to the complementary strand and disrupting its contact with the slower translocating domain(s) (Anderson and Kowalczykowski, 1997).

The DNA helicase activity requires the nucleotide cofactor adenosine triphosphate (ATP) and magnesium ion, with 1.7-3 molecules of ATP being hydrolyzed per base pair unwound. Helicase activity is inhibited by the ssDNA produced during processing of adsDNA molecule. Binding of SSB protein to the ssDNA products alleviates this inhibition (Anderson and Kowalczykowski, 1998).

In addition to being a helicase, the RecBCD enzyme is also a potent nuclease, functioning to protect the cell from invasion by infecting viral DNA. The RecBCD enzyme degrades both dsDNAand ssDNA, but the activity toward ssDNA is much lower than that for dsDNA. The dsDNA nuclease activity is coincident with translocation by RecBCD enzyme. Thus, although this degradation of dsDNA is formally defined as an 'exonuclease' activity, the cleavage is actually endonucleolytic and the requirement for a dsDNA end pertains to helicase function. Degradation during unwinding is asymmetric, with the 3 ' terminal strand (relative to the dsDNA entry site) being degraded much more vigorously than its complement. Hence the enzyme degrades dsDNA primarily in a 3 ' to 5 ' direction (Dixon and Kowalczykowski, 1993). SSB protein, in addition to binding the potentially inhibitory ssDNA produced by the helicase activity as discussed above, also moderates the 5' to 3' nuclease activity, lowering the frequency of cutting by the enzyme (Anderson and Kowalczykowski, 1998) (Table 1).

This voracious nuclease activity seems at odds with the fact that RecBCD enzyme plays 
a principal role in promoting DNA repair and homologous recombination, processes that, by their very nature, require the preservation of DNA. Resolution of this apparent inconsistency is found in the regulatory aspects of the recombination hotspot $x . x$ is a DNA locus that stimulates the frequency of genetic recombination in its vicinity. This recombination hotspot was originally discovered as a mutation in 1 phage that protected the phage genome from degradation by RecBCD enzyme (Lam et al., 1974). As shown in figure 4 , the sequence of $x$ is the octamer 5'-GCTGGTGG-3', and most single base mutations within the octamer reduce $x$ activity (Smith et al., 1981). Recombination in the vicinity of a $x$ site is stimulated by 5 - to 10 -fold over background levels. Key features need to be emphasized to understand the nature of this recombination hotspot.

First, the stimulation is highly polar, with the region of enhanced recombination extending downstream of the 5 ' end of the $\mathrm{w}$ sequence. Enhancement of recombination downstream of $w$ decreases by a factor of two for every $2.2-3.2 \mathrm{~kb}$, returning to background levels 10 $\mathrm{kb}$ downstream when no heterologous regions intervene (Myers et al., 1995). Second, all recombination stimulated by this site requires the activity of the RecBCD enzyme, whose nuclease activity would seemingly destroy its own substrate. Numerous genetic and biochemical studies have shown that the increase in recombination is due to a direct interaction between the $x$ sequence and the RecBCD enzyme, and that this stimulation only occurs if the enzyme approaches from the 3' side (Figure 4) (Taylor and Smith, 1995). The interaction with $x$ elicits several changes in enzyme function that are manifest in an overall decrease in nuclease activity, which accounts for the protection of DNA observed in vivo. Thus, $x$ is a regulator of RecBCD enzyme and, hence, of genetic recombination.

\section{Synapsis and invasion of single strands}

The pairing of the two recombining DNA molecules (synapsis) and invasion of a single strand from the initiating duplex into the other duplex are both catalyzed by the multifunctional protein $\operatorname{Rec} A$ invasion of the duplex DNA by a single stranded DNA results in the replacement of one of the strands of the original duplex with the invading strand, and the replaced strand is displaced from the duplex. Hence this reaction can also be called strand assimilation or strand exchange (Zaitsev and Kowalczykowski, 2000).

The process of single-strand assimilation occurs in three steps (Fig. 5). First, RecA polymerizes onto single-stranded DNA in the presence of ATP to form the presynaptic filament. The single strand of DNA lies within a deep groove of the RecA protein, and many RecA-ATP molecules coat the singlestranded DNA. One molecule of the RecA protein covers 3 to 5 nucleotides of singlestranded DNA. Next, the presynaptic filament aligns with homologous regions in the duplex DNA. The aligned duplex and single strand forms a paranemic joint, meaning that the single strand is not intertwined with the double strand at this point. Finally, the strands are exchanged from to form a plectonemic joint. In this stage, the invading single strand is now intertwined with the complementary strand in the duplex, and one strand of the invaded duplex is now displaced. In E. coli, exchange occurs in a $5^{\prime}$ to $3^{\prime}$ direction relative to the single strand and requires ATP hydrolysis (Arnold and Kowalczykowski, 2000).In contrast, the yeast homolog, Rad51, causes the single-strand to invade with the opposite polarity, i.e. 3' to 5'. Thus the direction of this polarity is not a universally conserved feature of recombination mechanisms. The product of strand assimilation is a heteroduplex in which one strand of the duplex was the original single- 
stranded DNA. The other strand of the original duplex is displaced (Fig. 6).

\section{Branch migration}

The movement of a Holliday junction to generate additional heteroduplex requires two proteins. One is the RuvA tetramer, which recognizes the structure of the Holliday junction. RuvB is an ATPase. It forms hexameric rings that provide the motor for branch migration. RuvA tetramers recognize the Holliday junction, and RuvB uses the energy of ATP hydrolysis to unwind the parental duplexes and form heteroduplexes between them (Iwasaki et al., 1992).

\section{Resolution}

Ruv $\mathrm{C}$ is the endonuclease that cleaves the Holliday junctions. It forms dimers that bind to the Holliday junction; recent data indicate an interaction among RuvA, RuvB and RuvC as a complex at the Holliday junction. The structure of the RuvA-Holliday junction complex suggests that the open structure of the junction stabilized by the binding of RuvA may expose a surface that is recognized by Ruv C for cleavage. RuvC cleaves symmetrically, in two strands with the same nearly identical sequences, thereby producing ligatable products. The preferred site of cleavage by RuvC is 5' WTT'S, where $\mathrm{W}=\mathrm{A}$ or $\mathrm{T}$ and $\mathrm{S}=\mathrm{G}$ or $\mathrm{C}$, and is the site of cleavage. RuvC can cut strands for either horizontal or vertical resolution. Strand choice is influenced by the sequence preference and also by the presence of RecA protein, which favors vertical cleavage (i.e. to cause recombination of flanking markers) (Dunderdale et al., 1991).

\section{Molecular basis of recombination in eukaryotes}

Homologous recombination (HR) serves to eliminate deleterious lesions, such as double- stranded breaks and inter strand cross links, from chromosomes. HR is also critical for the preservation of replication forks, for telomere maintenance, and chromosome segregation in meiosis I. The HR reaction is mediated by a conserved class of enzymes termed recombinases. Two recombinases, $\operatorname{Rad} 51$ and Dmc1, catalyze the pairing and shuffling of homologous DNA sequences in eukaryotic cells via a filamentous intermediate on ssDNA called the presynaptic filament. The assembly of the presynaptic filament is a ratelimiting process that is enhanced by recombination mediators, such as the breast tumor suppressor BRCA2. HR accessory factors that facilitate other stages of the Rad51- and Dmc1-catalyzed homologous DNA pairing and strand exchange reaction have also been identified (Bullard, 1996). The enzymes that mediate the pairing and shuffling of DNA sequences during HR are called recombinases, and the reaction mediated by these enzymes is termed homologous DNA pairing and strand exchange. Two recombinases, Rad51 and Dmc1, exist in eukaryotes. Rad51 is needed for mitotic HR events such as DSB repair and also for meiotic HR, whereas Dmc1 is only expressed in meiosis so its function is restricted therein. The salient attributes of the DMC1 gene and encoded protein are discussed in a separate section. Much of our knowledge on the RAD51 gene and its encoded protein has been derived from genetic and biochemical studies done in $S$. cerevisiae (Jimura et al., 1992).

The $S$. cerevisiae rad51 mutants are highly sensitive to DNA damaging agents and show defects in mitotic and meiotic recombination. Analysis of the $S$. cerevisiae RAD51 gene, which was cloned independently by three different groups, revealed significant homology of its encoded protein to the bacterial recombinase RecA, with particular conservation of those RecA residues that are critical for its recombinase function, including 
DNA binding and ATP hydrolysis. The structure of the $\operatorname{Rad} 51$ protein has been conserved among eukaryotes. Whereas $S$. cerevisiae rad51 mutants are viable mitotically. Just as in the case of RecA, with ATP (or an analogue of ATP) available, $S$. cerevisiae $\operatorname{Rad} 51$ protein assembles onto ssDNA or dsDNA to form a right-handed helical polymer that can span thousands of bases or base pairs. The Rad51-ssDNA nucleoprotein filament is often referred to as the presynaptic filament, and the biochemical steps that lead to the assembly of the $\operatorname{Rad} 51$ filament are collectively known as the presynaptic stage. Once assembled, the presynaptic filament captures a duplex DNA molecule and searches for homology in the latter. From studies done with RecA, it is expected that the homology search process occurs by way of random collisions between the presynaptic filament and the duplex molecule.

Thus, segments of the duplex are bound and tested in a reiterative fashion until homology is found. Upon the location of homology in the duplex molecule, the presynaptic filament is able to form DNA joints that are either "paranemic" or "plectonemic" in nature. In the paranemic joint, an internal region of the ssDNA is paired with the duplex molecule via canonical WatsonCrick hydrogen bonds, but the paired DNA strands are not topologically linked. The threestranded, paranemically paired nucleoprotein intermediate is referred to as the synaptic complex. Although relatively short-lived, the paranemic joint facilitates the location of a free DNA end to initiate the formation of a plectonemic joint, in which the participant DNA strands are bound by Watson-Crick hydrogen bonds and topologically intertwined. The nascent plectonemicjoint can be extended by DNA strand exchange being catalyzed by the presynaptic filament (Cao et al., 1990). The DNA strand exchange reaction is facilitated by the Rad54 protein. Moreover, Rad54 also promotes a specialized form of DNA strand exchange that involves the formation of a $\mathrm{HJ}$ and migration of the branch point in the HJ. Nucleation of Rad51 onto ssDNA is a slow process, which renders presynaptic filament assembly prone to interference by the ssDNA binding protein RPA. Certain recombinase accessory factors, which have been termed recombination mediators and include the tumor suppressor BRCA2, can overcome the inhibitory effect of RPA on the assembly of the Rad51 presynaptic filament. As such, these recombination mediators are critical for the efficiency of HR in vivo.

\section{The meiosis-specific recombinase DMC1}

The DMC1 gene was isolated by Bishop et al. in a screen for cDNA species specific for $S$. cerevisiae meiosis. The DMC1-encoded protein is present in almost all eukaryotes including humans and is structurally related to RecA and Rad51. Ablation of DMC1 in $S$. cerevisiae, Arabidopsis thaliana, and mice produces a constellation of meiotic abnormalities that reflect an indispensable role of the Dmc1 protein in meiotic recombination and chromosome segregation.

Dmc1 exists as an octamer in solution, and recent biochemical studies have provided compelling evidence that it too forms righthanded, helical filaments on ssDNA in an ATP-dependent manner and catalyze the homologous DNA pairing and strand exchange reaction within the context of these nucleoprotein filaments. Thus, in its action as a recombinase, Dmc1 possesses the same functional attributes as have been documented for RecA and Rad51 (Bishop, 1992).

The S. cerevisiae RAD52 Protein and its Recombination Mediator Activity

The $S$. cerevisiae Rad52 protein has been the most intensely studied recombination mediator to date. 
Table.1 Proteins and sites involved in genetic recombination in E. coli

\begin{tabular}{|c|c|}
\hline Protein and Site & Activity \\
\hline RecA & $\begin{array}{l}\text { DNA strand exchange, DNA renaturation, DNA-dependent } \\
\text { ATPase, DNA-and ATP- dependent co-protease }\end{array}$ \\
\hline RecBCD(exonuclease $\mathrm{V})$ & $\begin{array}{l}\text { DNA helicase, ATP-dependent dsDNA and ssDNA exonuclease, } \\
\text { ATP- stimulated ssDNA endonuclease, } \mathrm{x} \text { hotspot recognition }\end{array}$ \\
\hline RecBC & DNA helicase \\
\hline RecE (exonucleaseVIII) & dsDNA exonuclease, 5'-3'specific \\
\hline RecF & ssDNA dsDNA binding, ATP binding \\
\hline RecG & Branch migration of Holiday junctions, DNA helicase \\
\hline RecJ & ssDNA exonuclease, 5'-3' specific \\
\hline RecN & Unknown, ATP- binding consensus sequence \\
\hline $\mathrm{RecO}$ & Interaction with RecR and (possibly) RecF proteins \\
\hline RecQ & DNA helicase \\
\hline RecR & Interaction with $\mathrm{RecO}$ and (possibly) RecF proteins \\
\hline RecT & DNA renaturation \\
\hline RuvA & $\begin{array}{l}\text { Holiday-,cruciform-, and four-way junction binding; interaction } \\
\text { with RuvB proteins }\end{array}$ \\
\hline RuvB & $\begin{array}{l}\text { Branch migration of Holiday junctions, DNA helicase, } \\
\text { interaction with RuvA proteins }\end{array}$ \\
\hline RuvC & Holiday junction cleavage, four-way junction binding \\
\hline SbcB (exonuclease I) (xonA) & ssDNA exonuclease, 3'-5'specific, deoxyribophosphodiesterase \\
\hline SbcCD & ATP-dependent dsDNA exonuclease \\
\hline SSB & ssDNA binding \\
\hline DNA topoisomeraseI (topA) & $\varpi$ protein, type I topoisomerase \\
\hline DNAgyrase (gyrA and $g y r \mathrm{~B})$ & DNA gyrase, typeII topoisomerase \\
\hline DNA ligase (lig) & DNA ligase \\
\hline DNA polymerase I (polA) & DNA polymerase, 5'-3' exonuclease, 3'-5' exonuclease \\
\hline $\begin{array}{l}\text { Helicase II (uvrD, uvrE, recL, } \\
m u t \mathrm{U})\end{array}$ & DNA helicase \\
\hline Helicase IV (helD) & DNA helicase \\
\hline
\end{tabular}

\footnotetext{
${ }^{\mathrm{a}} \mathrm{sbcA}$ mutations are regulatory mutations that activate recE function.
} 
Fig.1 Holliday model for general recombination: single strand invasion
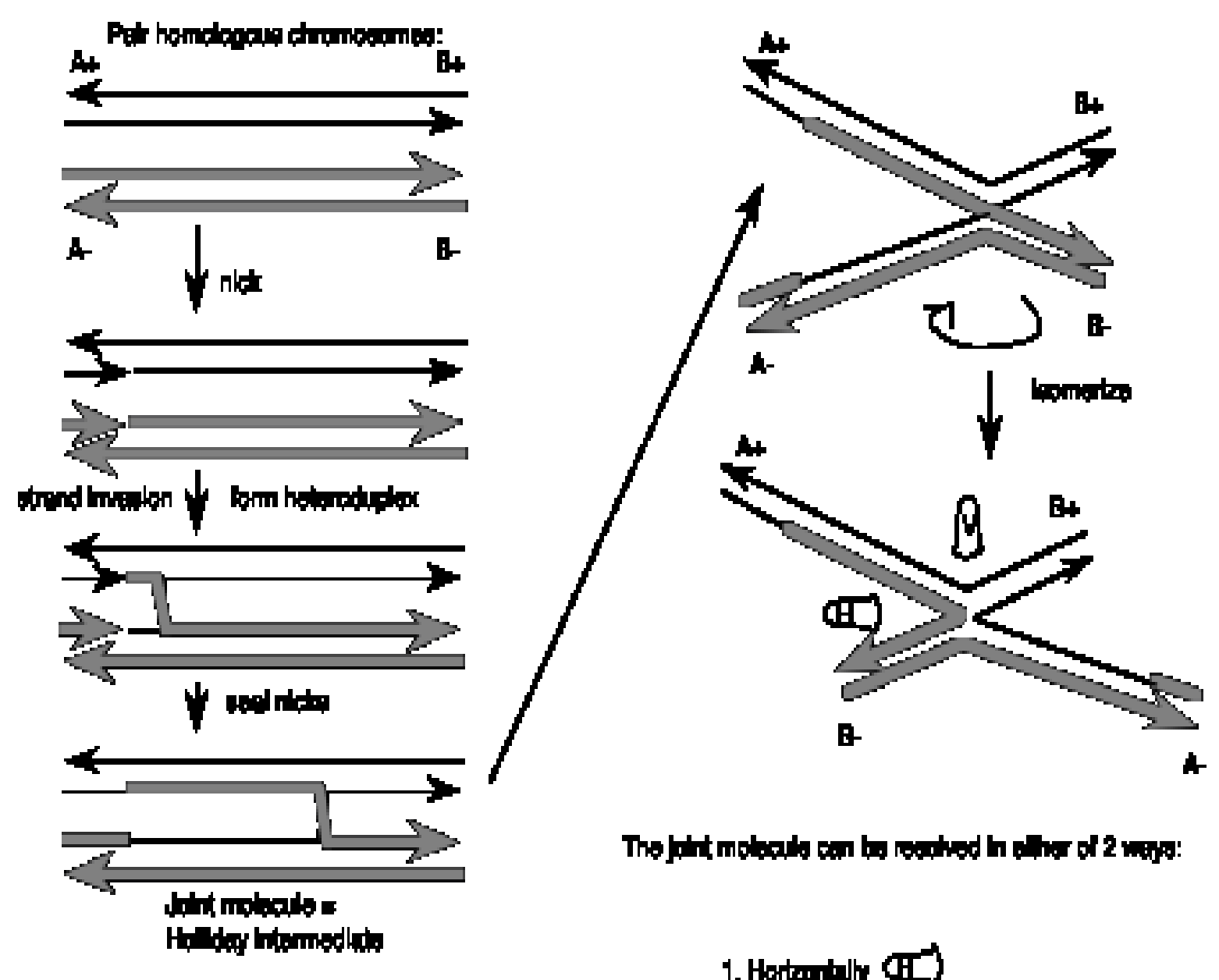

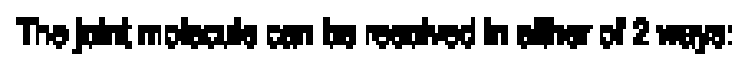

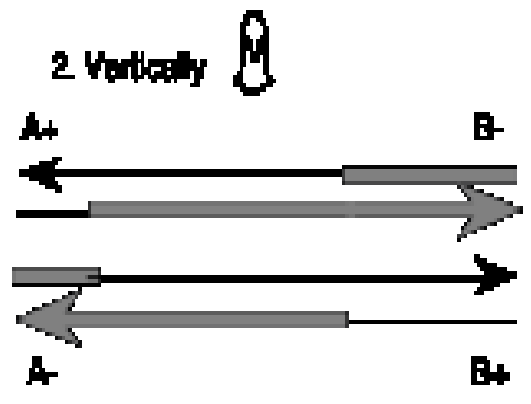

\section{Hontuindy}

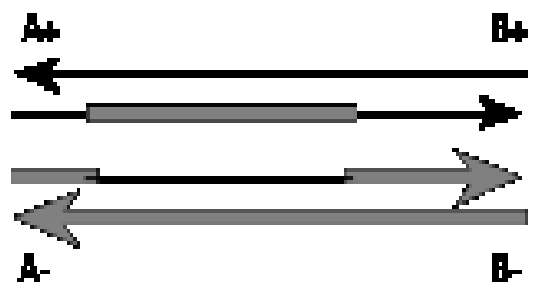

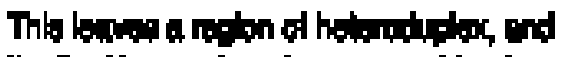

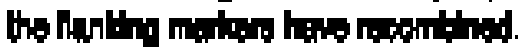

D月

A-

E-

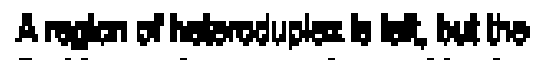

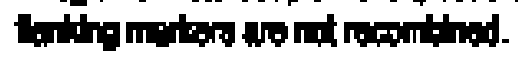


Fig.2 Double-strand-break model for recombination
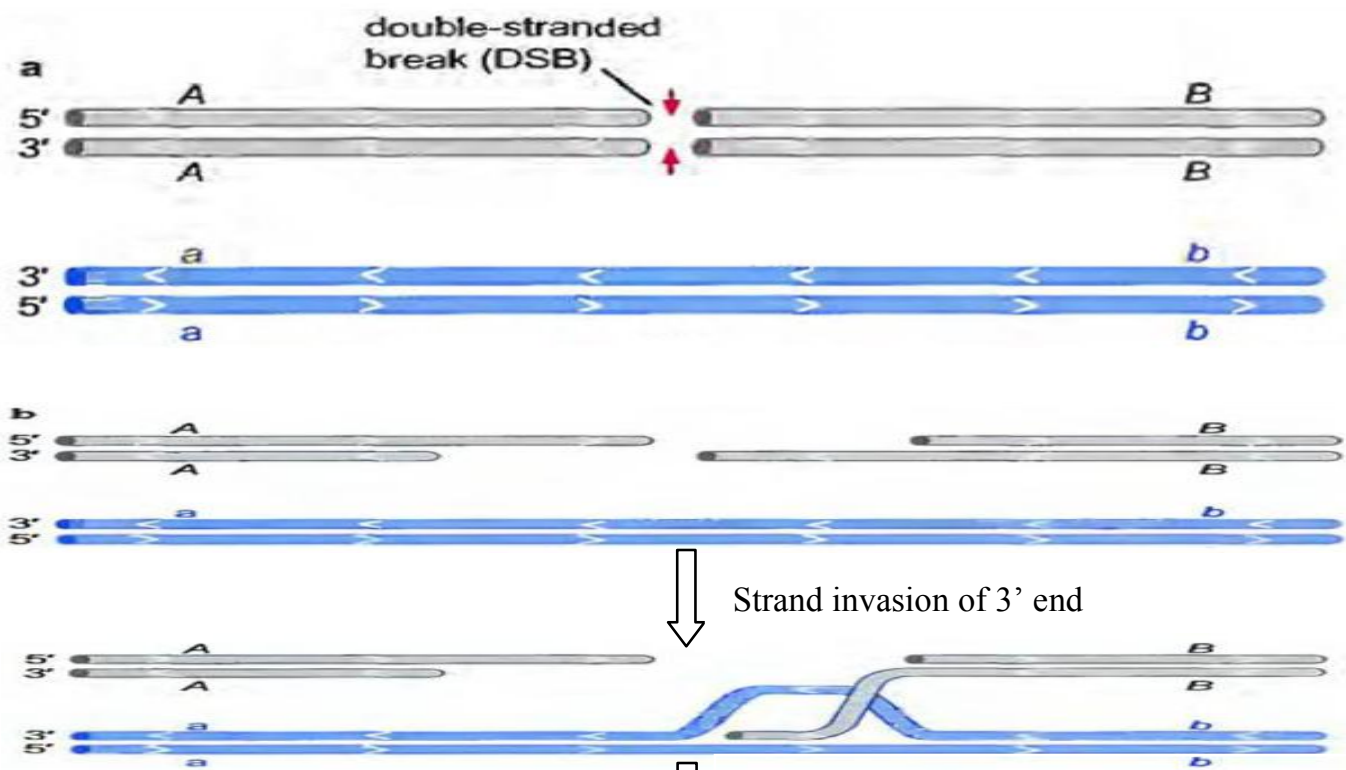

Second strand invasion and DNA repair synthesis at 3 ' end

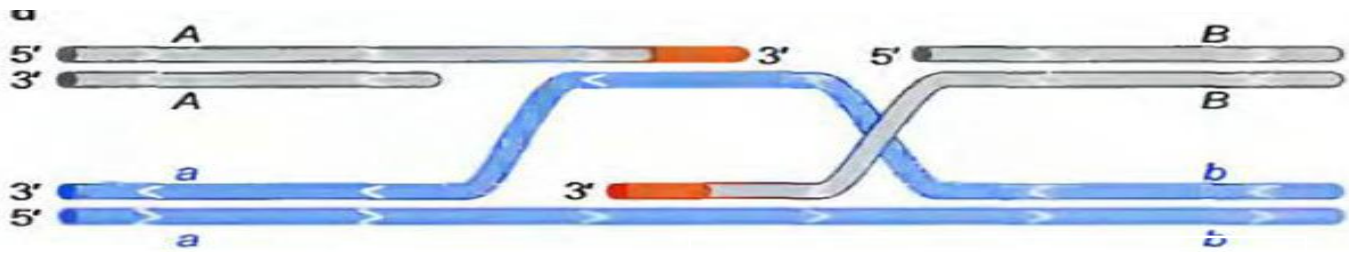

Branch migration and intermediate with two holiday junctions

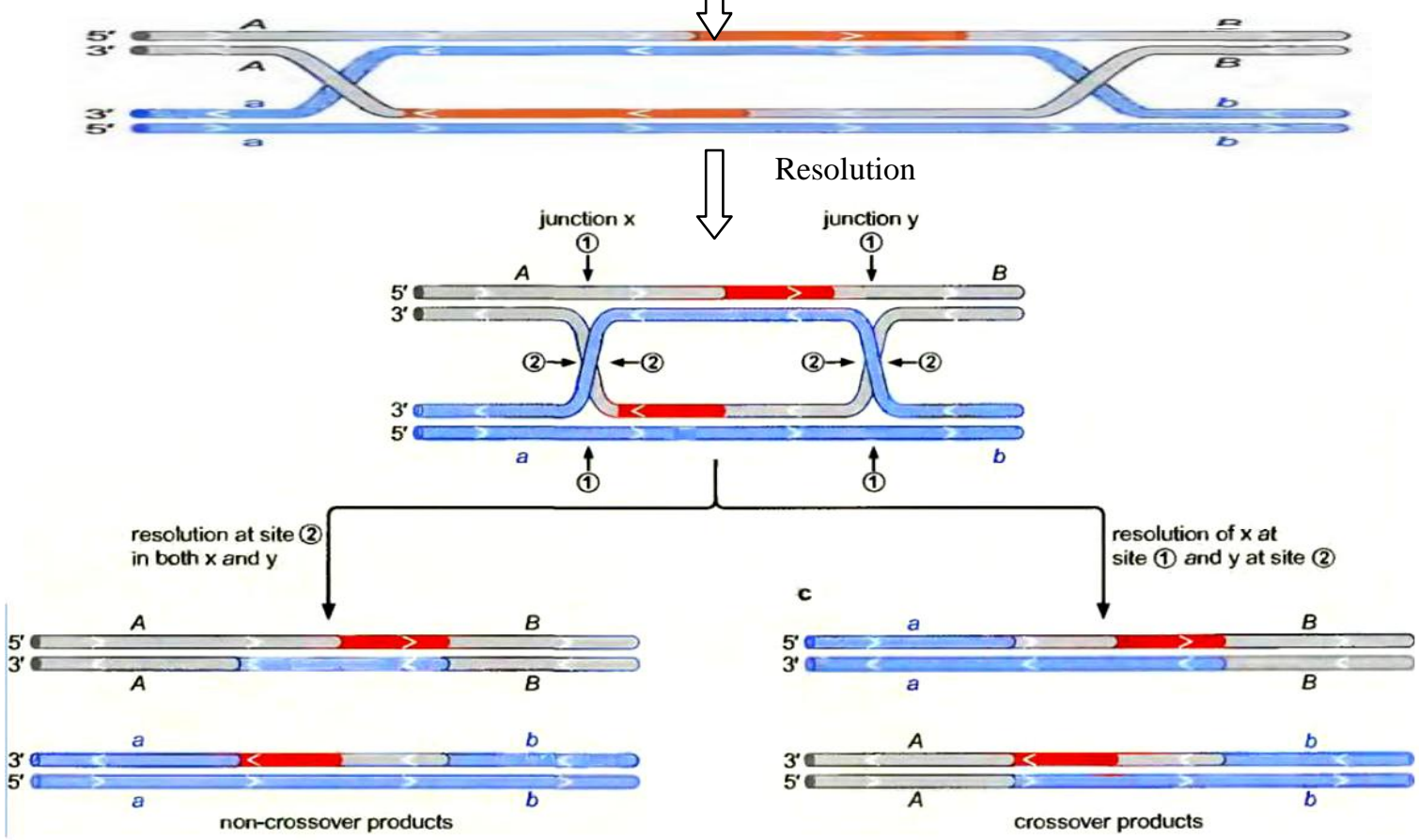


Fig.3 Initiation of homologous recombination by the coordinated activities of RecBCD enzyme and RecA protein

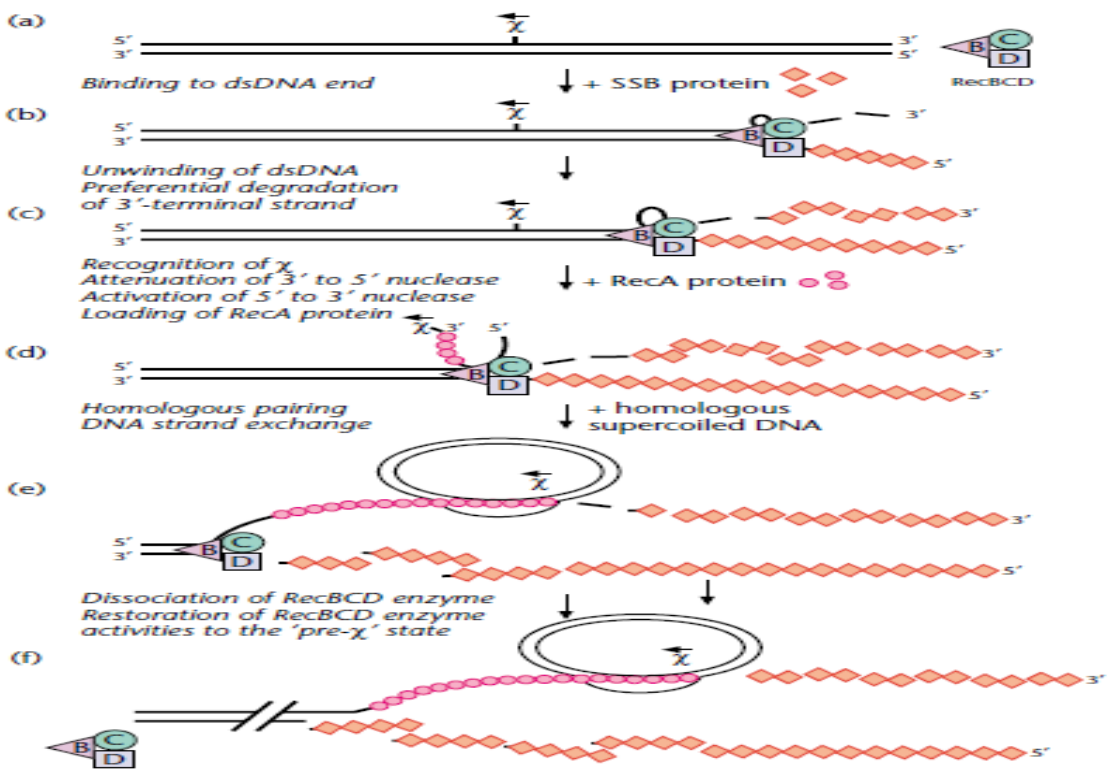

Fig.4 Orientation dependence of xrecognition

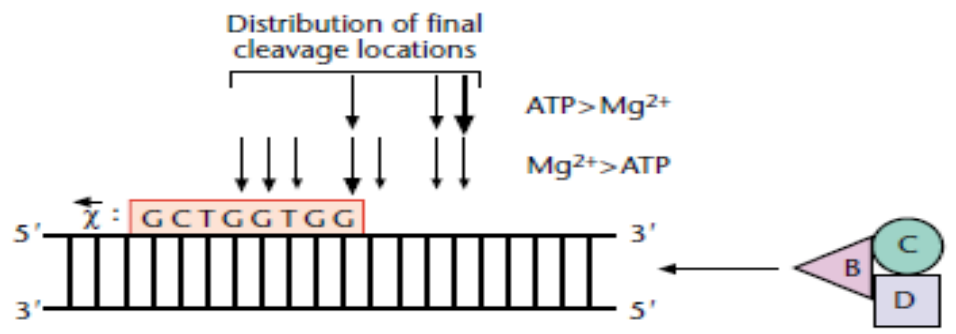

Fig.5 Role of RecA in assimilation of single-stranded DNA

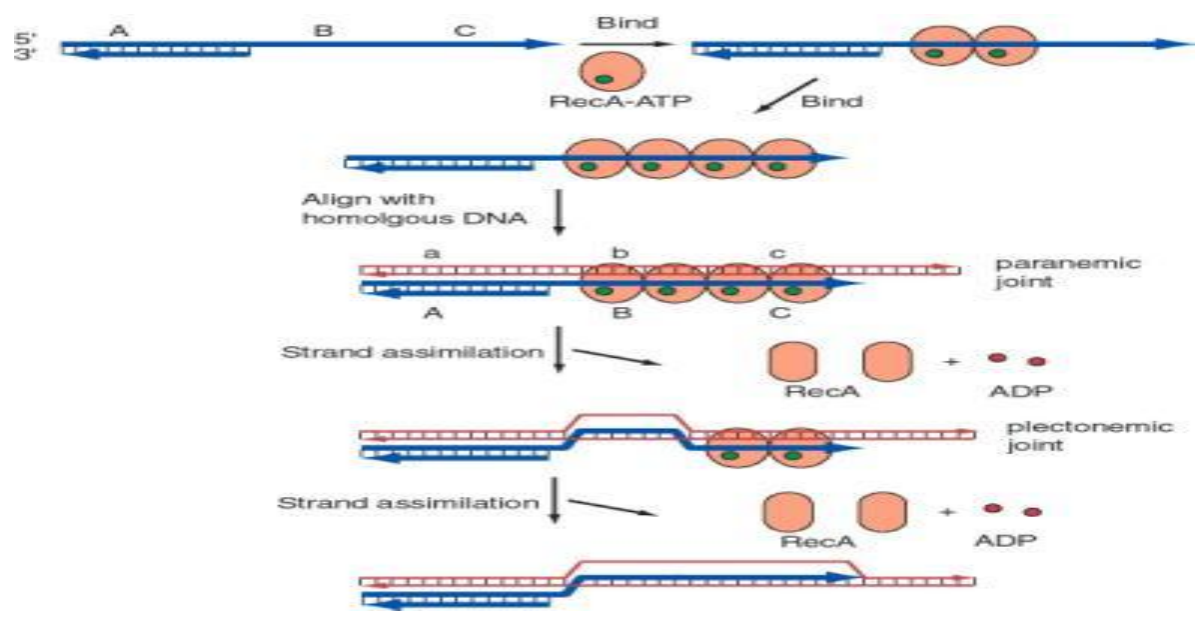


Fig.6 Schematic representation of recombination in eukaryotes

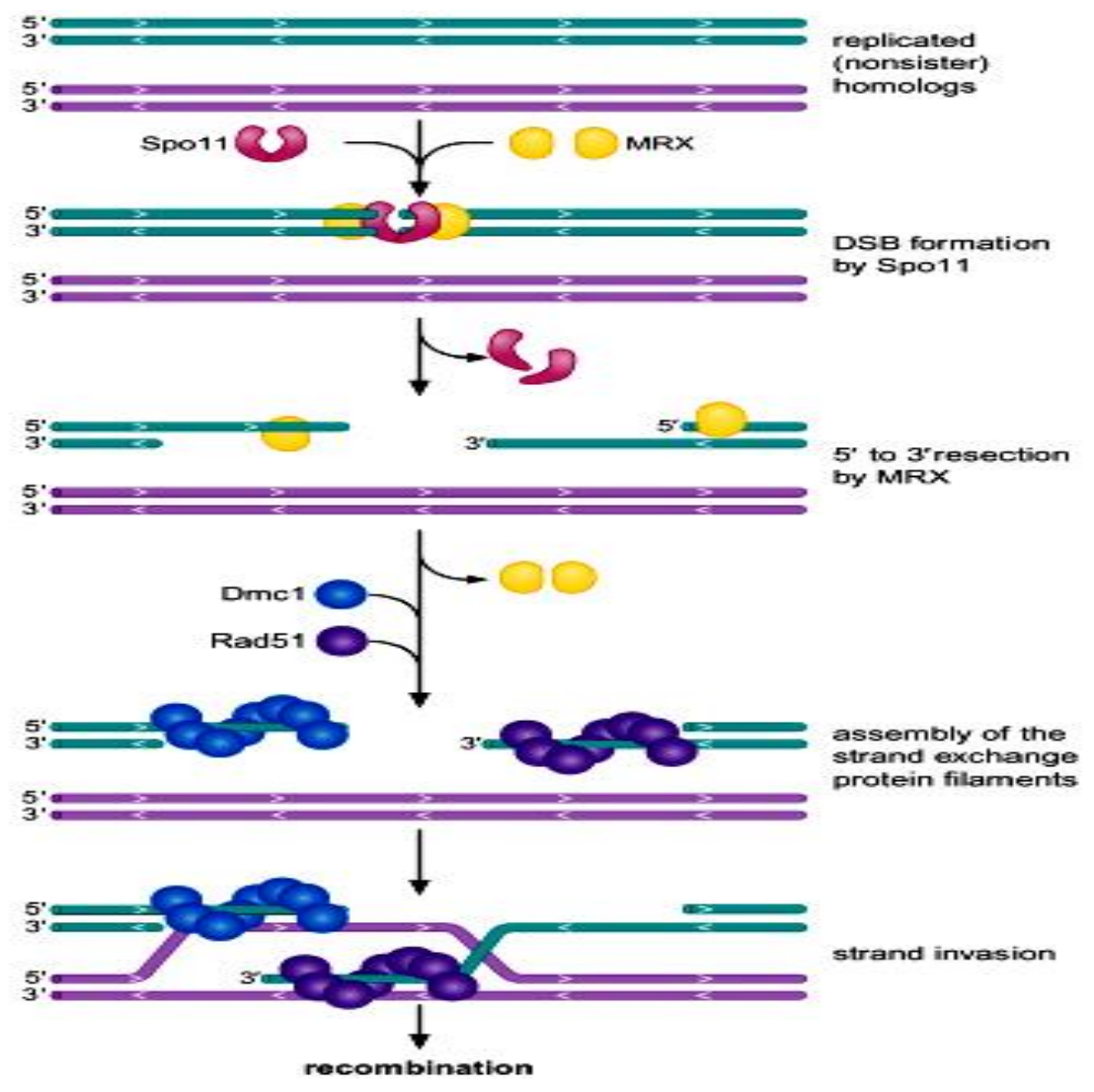

In both mitotic and meiotic cells, the recruitment of Rad51 to DSBs is strongly dependent on Rad52, but the DSB recruitment of Rad52 shows no dependence on Rad51. Taken together, the genetic and biochemical studies on $S$. cerevisiae $\operatorname{Rad52}$ provide compelling evidence that it helps deliver Rad51 to the ssDNA substrate during HR (Alani et al., 1990).

\section{Spo11 and the Formation of DNA Double- Strand Breaks in Meiosis}

Most sexually reproducing organisms use recombination to connect homologous paternal and maternal chromosomes to one another during prophase I of meiosis. This connection is essential for accurate chromosome segregation at the first meiotic division. Meiotic recombination has at its heart the formation and subsequent repair of
DNA double-strand breaks (DSBs) The major steps along the recombination pathway have been best defined in the budding yeast Saccharomyces cerevisiae (Keeney et al., 1997).DSB formation is catalyzed by Spo11, which appears to act via a topoisomerase-like reaction to generate a transient, covalent protein-DNA intermediate. After DSBs are formed, Spo11 is removed from the DNA and the $5^{\prime}$ strand termini are nucleolytically resected to yield variablelength, 3' single-stranded tails (Klapholz et $a l .$, 1985). In a series of reactions dependent on yeast homologs of bacterial RecA, these tails undergo strand invasion of intact homologous duplexes, ultimately giving rise to mature recombinant products. The repair of any given meiotic DSB can result in either reciprocal exchange of the chromosome arms flanking the break (a crossover), or no 
exchange of flanking arms (a non crossover or parental configuration).

In conclusion, general recombination allows large sections of the DNA double helix to move from one chromosome to another, and it is responsible for the crossing over of chromosomes that occurs during meiosis in fungi, animals, and plants. General recombination is essential for the maintenance of chromosomes in all cells, and it usually begins with a double-strand break that is processed to expose a single-stranded DNA end. Synapsis between this single strand and a homologous region of DNA double helix is catalyzed by the bacterial RecA protein and its eukaryotic homologs, and it often leads to the formation of a four-stranded structure known as Holiday junction. Depending on the pattern of strand cuts made to resolve this junction into two separate double helices, the products can be either a precisely repaired double-strand break or two chromosomes that have crossed over. Because general recombination relies on extensive basepairing interactions between the strands of the two DNA double helices that recombine, it occurs only between homologous DNA molecules. Gene conversion, the nonreciprocal transfer of genetic information from one chromosome to another, results from the mechanisms of general recombination, which involve a limited amount of associated DNA synthesis.

\section{Acknowledgement}

I am highly grateful to DST INSPIRE Fellowship awarded to me to carry out research. My sincere thanks are extended to DST for the timely disbursal of the grant.

\section{References}

Alani, E., Padmore, R., Kleckner, N. 1990. Analysis of wild type and rad50 mutants of yeast suggests an intimate $\begin{array}{lcr}\begin{array}{l}\text { relationship } \\ \text { chromosome }\end{array} & \text { between } & \text { meiotic } \\ \text { recombination. Cell, 61: 419-436. } & \text { and }\end{array}$

Anderson, D.G. and Kowalczykowski, S.C. 1997. The translocating RecBCD enzyme stimulates recombination by directing RecA protein onto ssDNA in a x-regulated manner. Cell, 90: 77-86.

Anderson, D.G. and Kowalczykowski, S.C. 1998. SSB protein controls RecBCD enzyme nuclease activity during unwinding: a new role for looped intermediates. J. Mol. Biol., 282: 275285.

Arnold, D.A. and Kowalczykowski, S.C. 2000. Facilitated loading of RecA protein by RecBCD enzyme is essential to recombination. J. Biol. Chem., (in press).

Bishop, D.K., Park, D., Xu, L. and Kleckner, N., 1992. DMC1: a meiosis-specific yeast homolog of $\mathrm{E}$. coli $\mathrm{rec} A$ required for recombination, synaptonemal complex formation, and cell cycle progression. Cell, 69: 439-456.

Bullard, S.A., Kim, S., Galbraith, A.M., Malone, R.E. 1996. Double strand breaks at the HIS2 recombination hot spot in Saccharomyces cerevisiae, Proc. Natl. Acad. Sci. USA, 93: 13054-13059.

Cao, L., Alani, E. and Kleckner, N.1990. A pathway for generation and processing of double-strand breaks during meiotic recombination in S. cerevisiae. Cell, 6: 1089-1101.

Dixon, D.A. and Kowalczykowski, S.C. 1993. The recombination hotspot $\mathrm{W}$ is a regulatory sequence that acts by attenuating the nuclease activity of the E. coli Rec BCD enzyme. Cell, 73: 8796.

Dunderdale, H.J., Benson, F.E., Parsons, C.A., Sharples, G.J., Lloyd, R.G. and West, S.C. 1991. Formation and resolution of recombination intermediates by E. coli RecA and 
RuvC proteins. Nature, 354: 506-510.

Iwasaki, H., Takahagi, M., Nakata, A., and Shinagawa, H. 1992. Escherichia coli RuvA and RuvB proteins specifically interact with Holliday junctions and promote branch migration. Genes Dev., 6: 2214-2220.

Jimura, M., Leem, S.H., Ogawa, H. 1992. Identification of new genes required for meiotic recombination in Saccharomyces cerevisiae. Genetics, 133:51-66.

Keeney, S., Giroux, C.N. and Kleckner, N. 1997. Meiosis- specific DNA doublestrand breaks are catalyzed by Spo11, a member of a widely conserved protein family. Cell, 88: 375-384.

Klapholz, S., Waddell, C.S. and Esposito, R.E. 1985. The role of the SPO11 gene in meiotic recombination in yeast. Genetics, 110: 187-216.

Kowalczykowski, S.C., Dixon, D.A., Eggleston, A.K., Lauder, S.D. and Rehrauer, W.M. 1994. Biochemistry of homologous recombination in Escherichia coli. Microbiol. Rev., 58: 401-465.

Lam, S.T., Stahl, M.M., McMilin, K.D. and Stahl, F.W.1974. Rec-mediated recombinational hot spot activity in bacteriophage lambda. II. A mutation which causes hot spot activity.
Genetics, 77: 425-433.

Myers, R.S., Stahl, M.M. and Stahl, F.W. 1995. $x$ recombination activity in phage 1 decays as a function of genetic distance. Genetics, 141: 805-812.

Roman, L.J., Eggleston, A.K. and Kowalczykowski, $\quad$ S.K. 1992. Processivity of the DNA helicase activity of Escherichia coli Rec BCD enzyme. J. Biol. Chem., 267: 42074214.

Smith, G.R., Kunes, S.M., Schultz, D.W., Taylor, A. and Triman, K.L.1981. Structure of Chi hotspots of generalized recombination. Cell, 24: 429-436.

Szostak, J.W., Orr-Weaver, T.L., Rothstein, R.J. and Stahl, F.W. 1983. The doublestrand break repair model for recombination. Cell, 33: 25-35.

Taylor, A. and Smith, G.R. 1980. Unwinding and rewinding of DNA by the recBC enzyme. Cell, 22: 447-457.

Taylor, A.F. and Smith, G.R. 1995. Strand specificity of nicking of DNA at Chi sites by RecBCD enzyme. Modulation by ATP and magnesium levels. J. Biol. Chem., 270: 24459-24467.

Zaitsev, E.N. and Kowalczykowski, S.C. 2000. A novel pairing process promoted by Escherichia coli RecA protein: Inverse DNA and RNA strand exchange. Genes Dev. (in press).

\section{How to cite this article:}

Mamta Nehra, Rajesh Kumar Sharma and Mukesh Choudhary. 2017. An Overview on Molecular Basis of Genetic Recombination. Int.J.Curr.Microbiol.App.Sci. 6(4): 1154-1167. doi: https://doi.org/10.20546/ijcmas.2017.604.142 